OPEN ACCESS

Edited by:

Giuseppe Lombardi,

Veneto Institute of Oncology

(IRCCS), Italy

Reviewed by:

Ghazaleh Tabatabai,

Tübingen University Hospital,

Germany

Veronica Villani,

Regina Elena National Cancer Institute

(IRCCS), Italy

*Correspondence:

Junping Zhang

doczhjp@hotmail.com

Specialty section:

This article was submitted to

Neuro-Oncology and

Neurosurgical Oncology,

a section of the journal

Frontiers in Oncology

Received: 31 August 2020 Accepted: 02 December 2020 Published: 04 February 2021

Citation:

Ge J, Li C, Xue F, Qi S, Gao Z, Yu C and Zhang J (2021) Apatinib Plus Temozolomide: An Effective Salvage Treatment for

Recurrent Glioblastoma.

Front. Oncol. 10:601175. doi: 10.3389/fonc. 2020.601175

\section{Apatinib Plus Temozolomide: An Effective Salvage Treatment for Recurrent Glioblastoma}

\author{
Jingjing $\mathrm{Ge}^{1}$, Cheng $\mathrm{Li}^{1}$, Fengjun Xue ${ }^{1}$, Shaopei $\mathrm{Qi}^{1}$, Zhimeng Gao ${ }^{1}$, Chunjiang $Y u^{2}$ \\ and Junping Zhang ${ }^{1 *}$ \\ ${ }^{1}$ Department of Neuro-Oncology, Sanbo Brain Hospital, Capital Medical University, Beijing, China, ${ }^{2}$ Department of \\ Neurosurgery, Sanbo Brain Hospital, Capital Medical University, Beijing, China
}

Background: Treatment for recurrent glioblastoma is poor, and there is a need for better therapies. Here we retrospectively assessed the efficacy and toxicity of temozolomide plus apatinib, an oral small-molecule tyrosine kinase inhibitor targeting vascular endothelial growth factor receptor 2 in recurrent glioblastoma.

Materials and Methods: A retrospective analysis of patients with recurrent glioblastoma who underwent apatinib plus temozolomide treatment was performed. Apatinib was given at $500 \mathrm{mg}$ once daily. Temozolomide was administered at $200 \mathrm{mg} / \mathrm{m}^{2} / \mathrm{d}$ on days $1-5$ or $50 \mathrm{mg} / \mathrm{m}^{2} / \mathrm{d}$ continuous daily according to whether they had experienced temozolomide maintenance treatment before. The main clinical data collected included tumor characteristics, status of MGMT promoter, and IDH mutation, number of relapse, response, survival, adverse reactions, and salvage therapies.

Results: From April 2016 to August 2019, thirty-one patients were identified. The objective response rate was $26.3 \%$, and the disease control rate was $84.2 \%$. The progression-free survival (PFS) at 6 months and overall survival (OS) at 12 months were 44.6 and $30.2 \%$. The median PFS and OS were 4.9 and 8.2 months, respectively. Two patients achieved long PFS of 30.9 and 38.7+ months. The median survival time after progression of the patients with or without salvage bevacizumab was 5.1 versus 1.2 months. The most common grade 3 or 4 toxicities were hypertension (5.8\%), decreased appetite (5.8\%), and thrombocytopenia (4.3\%), most of which were resolved after symptomatic treatment or dose reduction.

Conclusion: Apatinib plus temozolomide is an effective salvage regimen with manageable toxicities for recurrent glioblastoma and could not reduce the sensitivity to bevacizumab.

Keywords: glioblastoma, apatinib, angiogenesis inhibitors, temozolomide, VEGFR-2, chemotherapy 


\section{INTRODUCTION}

Glioblastoma is the most common and aggressive group of primary central nervous system tumors (1). In 2005, Stupp and colleagues established a standard treatment for newly diagnosed glioblastoma consisting of concomitant chemoradiotherapy with temozolomide and then maintenance treatment with temozolomide for 6 to 12 months. However, even with current standard treatment, the median overall survival (OS) and progression-free survival (PFS) are only 14.6 and 6.9 months respectively for glioblastoma (2). Tumors ultimately recur, killing most of patients.

To our knowledge, no standard of care has been established for patients with recurrent glioblastoma. Besides histological hallmarks of anaplasia, cell proliferation, and necrosis, glioblastoma is characterized by marked microvascular proliferation and vascular permeability (3). Thus, antiangiogenesis is a promising therapeutic strategy and vascular endothelial growth factor (VEGF) signaling is one of the bestcharacterized key therapeutic targets.

The most extensively investigated regimen for recurrent glioblastoma is bevacizumab, a humanized monoclonal antibody that inhibits VEGF. Based on two prospective Phase II trials with non-inferior response rate and PFS rate at 6 months (6m-PFS) of $36 \%$, bevacizumab was approved by the US Food and Drug Administration for recurrent glioblastoma in 2009 (4, 5). In the EORTC 26101 phase 3 trial, for the glioblastoma with a first progression after standard chemoradiotherapy, added bevacizumab prolonged the median PFS, which was 4.2 months for the bevacizumab plus lomustine group and 1.5 months for the lomustine group (HR 0.49). The $6 \mathrm{~m}$-PFS was $30.2 \%$ for bevacizumab plus lomustine group and $16.9 \%$ for the lomustine group. However, the combined therapy did not confer a survival advantage over the monotherapy: 9.1 months versus 8.6 months (6). Moreover, there are no effective salvage therapies after bevacizumab failure (7).

In the VEGF signaling family, vascular endothelial growth factor receptor-2 (VEGFR-2) is the primary receptor that mediates angiogenesis (8). Apatinib, a small-molecule tyrosine kinase inhibitor that is administered orally, selectively binds to and strongly inhibits VEGFR-2. Apatinib was approved to treat patients with advanced gastric cancer refractory to two or more lines of prior chemotherapy in China (9). Several studies have revealed that apatinib also significantly improved the survival of intermediate and advanced hepatocellular carcinoma (10), ovarian cancer (11), and advanced non-squamous non-small cell lung cancer (12). In addition, apatinib can reverse multidrug resistance and enhance the efficacy of some conventional anticancer drugs, such as doxorubicin, vincristine, and verapamil $(13,14)$. In a preclinical study, apatinib suppressed glioma cell growth and metastasis and promoted anti-tumor activity of temozolomide (15).

In this study, we investigated the efficacy and safety of apatinib in combination with temozolomide in patients with recurrent glioblastoma.

\section{MATERIALS AND METHODS}

\section{Patients}

We performed a retrospective study for all the patients with recurrent glioblastoma treated with apatinib plus temozolomide in Sanbo Brain Hospital, Capital Medical University between April 2016 and August 2019. This study was approved by the Ethics Committee of Sanbo Brain Hospital, Capital Medical University.

Inclusion criteria were as follows: Age at 18-70 years old; Karnofsky Performance Score (KPS) $\geq 50 \%$; histological diagnosis of glioblastoma; recurrence was confirmed histologically or by radiographic evidence; no previous treatment with bevacizumab or other antiangiogenic drugs; treated with at least one cycle of the combined regimen of apatinib and temozolomide; had at least one post-treatment radiographic follow-up. All of the patients were required to have normal hematologic, hepatic and renal function to be eligible for treatment. Patients previously use enzyme-inducing antiepileptic drugs (EIAEDs) had been switched to non-EIAEDs at least 2 weeks before treatment. Patients with incomplete medical records were excluded.

\section{Treatment}

All patients received oral apatinib $500 \mathrm{mg}$ once daily in combination with temozolomide. Temozolomide was administered at $200 \mathrm{mg} /$ $\mathrm{m}^{2} / \mathrm{d}$ according to the standard 5/28 days regimen for patients who had not previously received temozolomide. Patients who experienced a relapse following the standard 5/28 temozolomide schedule received continuous daily temozolomide $\left(50 \mathrm{mg} / \mathrm{m}^{2} / \mathrm{d}\right)$. One treatment cycle was defined as 28 days (4 weeks). Patients continued treatment until they experienced disease progression or unacceptable toxicity. Patients provided informed consent authorizing the use of their personal information for research purposes before treatment.

The clinical data collected included the following: age, sex, KPS, previous therapies, tumor size, number of relapse, status of O6-methylguanine DNA-methyltransferase (MGMT) promoter and isocitrate dehydrogenase (IDH) mutation, treatment cycles, response, dose changes, adverse reactions, progression date, salvage therapies after progression, and dead date.

\section{Assessments}

The radiographic responses were classified according to the RANO criteria (16). Contrast-enhanced MRI was performed at baseline and every two cycles thereafter until disease progression. If complete response (CR) or partial response (PR) was achieved, MRI was conducted after the following cycle to confirm the efficacy. Diffusion and perfusion-weighted imagings were introduced to differentiate pseudoprogression from true progression. Toxicities were classified according to the Common Terminology Criteria for Adverse Events (CTCAE) 4.0.

\section{Statistical Analyses}

The endpoints include a $6 \mathrm{~m}$-PFS, objective response rate, median PFS, median OS, OS rate at 12 months (12m-OS) and toxicity. PFS was defined as the time interval from treatment initiation to disease progression, death from any cause, or last follow-up, 
whichever occurs first. OS was defined as the time interval from treatment initiation to death from any cause or last follow-up.

Statistical analyses were performed using SPSS 24.0 software. Categorical variables were described by numbers and percentages, and continuous variables were described by median and range. Survival curves for PFS and OS were analyzed using the KaplanMeier method. The log-rank test was used to evaluate relations between survival and categorical predictor variables (MGMT status, IDH status, number of relapse, tumor dissemination, bevacizumab use after progression). $P$ values of $<0.05$ were considered statistically significant.

\section{RESULTS}

\section{Patient and Disease Characteristics}

Between April 2016 and August 2019, a total of 31 patients were identified (Table 1). Twenty of the 31 patients (64.5\%) were men, and the median age was 53 years old (range, 21-70 years). Nine patients (29\%) had experienced two or more progressions before treatment. Seven patients just received radiotherapy concomitant with temozolomide, without maintenance treatment. Twentythree patients (74.2\%) experienced disease progression while on 5 -day temozolomide maintenance therapy, and one got progressed 35.5 months after temozolomide discontinuation. The median time interval of last recurrence and temozolomide discontinuation was 0.9 months (range, 0-35.5 months). Seventeen $(54.8 \%)$ patients had tumors with intracranial dissemination. Nineteen $(61.3 \%)$ patients had tumors with the maximal diameter larger than $30 \mathrm{~mm}$. Five patients received tumor total resection at the last relapse and had non-measurable lesion. Nineteen $(61.3 \%)$ patients had unmethylated MGMT promoter. Twenty (64.5\%) patients have IDH wild-type tumors.

\section{Response to Treatment}

The response to this combined treatment is shown in Figure 1. Of the 31 patients, three got $\mathrm{CR}$, five got $\mathrm{PR}, 17$ remained stable disease (SD) and six got progressed disease (PD). All of the five patients with non-measurable lesion achieved SD. The objective response rate (ORR) was $26.3 \%$, and the disease control rate (DCR) was $84.2 \%$. Figure 2 shows the MRI changes of one patient who got CR. After receiving two cycles of therapy, the extensive enhanced tumors were obviously diminished, and the non-enhanced lesions (Flair) were decreased (Figures 2A-H). The response was confirmed by MRI attained after three cycles of therapy (Figures 2I-L).

Of the 22 patients with first relapsed glioblastoma, three got $\mathrm{CR}$, four got PR, 11 got SD, and four got PD. The ORR was $31.8 \%$, and the DCR was $81.8 \%$.

\section{Efficacy}

The median follow-up period was 7.9 (range, 3.2 to 38.7) months. At the last follow-up (December 31, 2019), one patient was still under treatment and one was lost to follow-up after three evaluations.

For all of the patients, the estimated $6 \mathrm{~m}$-PFS rate was $44.6 \%$ (95\% CI, 27 to $62.2 \%$ ). The $12 \mathrm{~m}$-OS rate was $30.2 \%$ (95\% CI, 13.7
TABLE 1 | Baseline characteristics of patient and tumor.

\begin{tabular}{|c|c|}
\hline Characteristic & rGBM, $n=31$ \\
\hline \multicolumn{2}{|l|}{ Age, years } \\
\hline Mean & 51.5 \\
\hline Median & 53 \\
\hline Range & $21-70$ \\
\hline \multicolumn{2}{|l|}{ Sex, n(\%) } \\
\hline Male & $20(64.5)$ \\
\hline Female & $11(35.5)$ \\
\hline \multicolumn{2}{|l|}{ Initial KPS, n(\%) } \\
\hline $90-100$ & $10(32.3)$ \\
\hline $70-80$ & $12(38.7)$ \\
\hline $50-60$ & $9(29.0)$ \\
\hline \multicolumn{2}{|l|}{ No. of relapse, n(\%) } \\
\hline First & $22(71.0)$ \\
\hline Second & $6(19.4)$ \\
\hline Third & $3(9.7)$ \\
\hline \multicolumn{2}{|l|}{ No. of previous surgery, n(\%) } \\
\hline 1 & $17(54.8)$ \\
\hline 2 & $9(29.0)$ \\
\hline 3 & $5(16.1)$ \\
\hline \multicolumn{2}{|l|}{ Resection at last relapse, n(\%) } \\
\hline Yes & $9(29.0)$ \\
\hline No & $22(71.0)$ \\
\hline \multicolumn{2}{|l|}{ Maximal diameter ${ }^{\mathrm{a}}, \mathrm{n}(\%)$} \\
\hline$>30$ mm & 19 (61.3) \\
\hline$\leq 30 \mathrm{~mm}$ & 7 (22.6) \\
\hline non-measurable lesion & $5(16.1)$ \\
\hline \multicolumn{2}{|l|}{ Tumor dissemination, n(\%) } \\
\hline No & $9(29.0)$ \\
\hline intracranial dissemination & $17(54.8)$ \\
\hline non-measurable lesion & $5(16.1)$ \\
\hline \multicolumn{2}{|l|}{ Previous chemotherapy, n(\%) } \\
\hline TMZ, concomitant & $29(93.5)$ \\
\hline TMZ, maintenance none & 7 (22.6) \\
\hline TMZ, maintenance, 5/28 & $24(77.4)$ \\
\hline Progression on TMZ, n(\%) & $23(74.2)$ \\
\hline Progression after TMZ, n(\%) & $1(3.2)$ \\
\hline Patient characteristics & rGBM, $n=31$ \\
\hline \multicolumn{2}{|c|}{ Median time of last recurrence [range], mo } \\
\hline From diagnosis & $10.8[2.3-43.8]$ \\
\hline From TMZ discontinuation & $0.9[0-35.5]$ \\
\hline \multicolumn{2}{|l|}{ MGMT status, n(\%) } \\
\hline Unmethylated & $19(61.3)$ \\
\hline Methylated & $5(16.1)$ \\
\hline Not done/unknown & 7 (22.6) \\
\hline \multicolumn{2}{|l|}{ IDH1/2 mutation, $\mathrm{n}(\%)$} \\
\hline No & $20(64.5)$ \\
\hline Yes & $2(6.5)$ \\
\hline Not done/unknown & $9(29.0)$ \\
\hline
\end{tabular}

rGBM, recurrent glioblastoma; KPS, Karnofsky performance status; TMZ, temozolomide; MGMT, O6-methylguanine DNA-methyltransferase; IDH, isocitrate dehydrogenase.

a Maximal tumor diameter determined on contrast enhanced axial, sagittal, or coronal Magnetic Resonance Imaging.

to $46.7 \%$ ). The median PFS was 4.9 months (95\%CI, 2.8 to 7 months). The median OS was 8.2 months (95\%CI, 6.9 to 9.5 months). The median time interval between disease progressions to death was 3.2 months. Figure 3 shows the Kaplan-Meier curves of PFS and OS of the whole population.

An exciting finding was that the two patients who discontinued treatment without tumor progression after 2 years achieved long PFS of 30.9 and 38.7+ months, while their prior recurrence intervals were only 12 and 14 months, respectively. Both of them harbored unmethylated MGMT and 


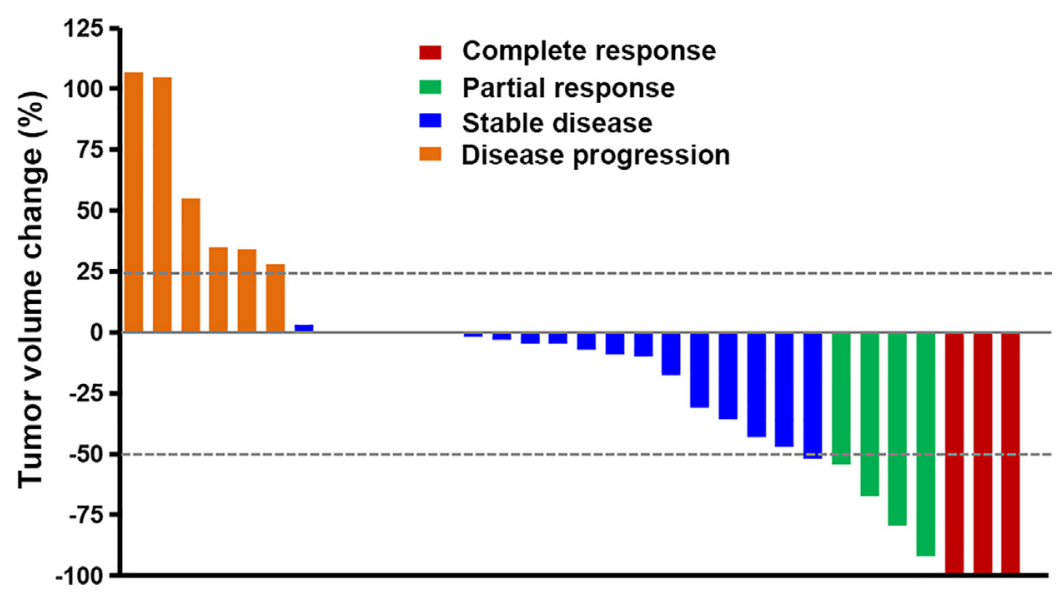

Patients

FIGURE 1 | The best response to apatinib plus temozolomide treatment. Five patients had non-measurable lesion and had 0\% change from baseline. The different colors indicate different responses. The response was classified according to the RANO criteria.
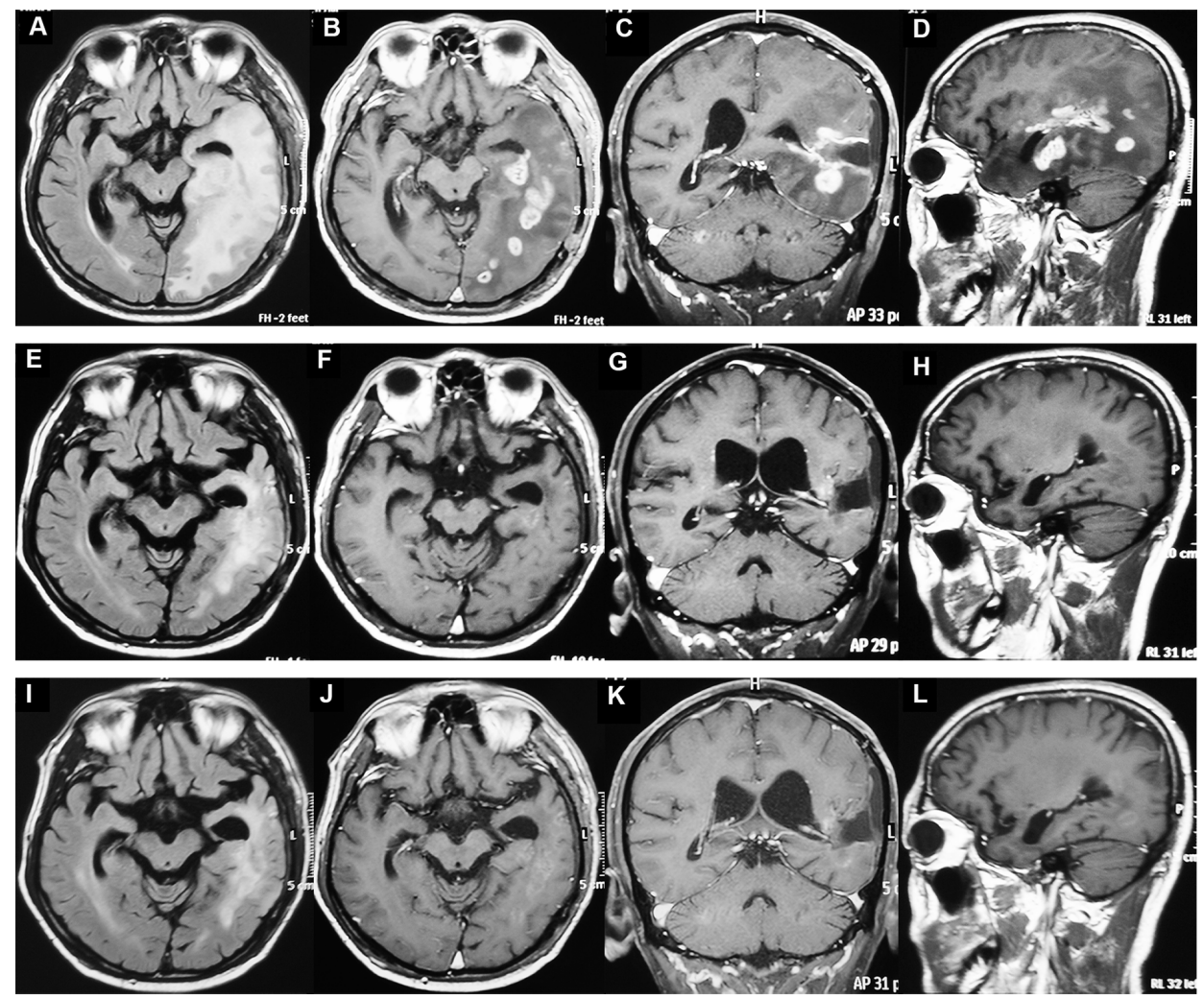

FIGURE 2 | MRI of one patient with recurrent glioblastoma before and after apatinib plus temozolomide treatment. (A-D) The MRI before treatment. The tumors were enhanced and intracranial disseminated. (E-H) The MRI after two cycles of treatment. The enhanced tumors disappeared and the non-enhanced lesions (Flair) were decreased. (I-L) MRI after three cycles of treatment. The patient had a complete response.

wild-type IDH. One received tumor total resection at the last relapse, and the other one had a local tumor without resection.

For the 20 patients with wild-type IDH, the median PFS and $6 \mathrm{~m}$-PFS were 5.7 months (95\%CI, 3.6 to 7.8 months) and $48.7 \%$, and the median OS and $12 \mathrm{~m}$-OS were 8.2 (95\%CI, 6.8 to 9.6 months) and $37.1 \%$. For the 24 patients who experienced disease progression while on 5-day temozolomide, median PFS was 5.7 months (95\%CI, 2.2 to 9.2 months) and OS was 8.5 months (95\% 

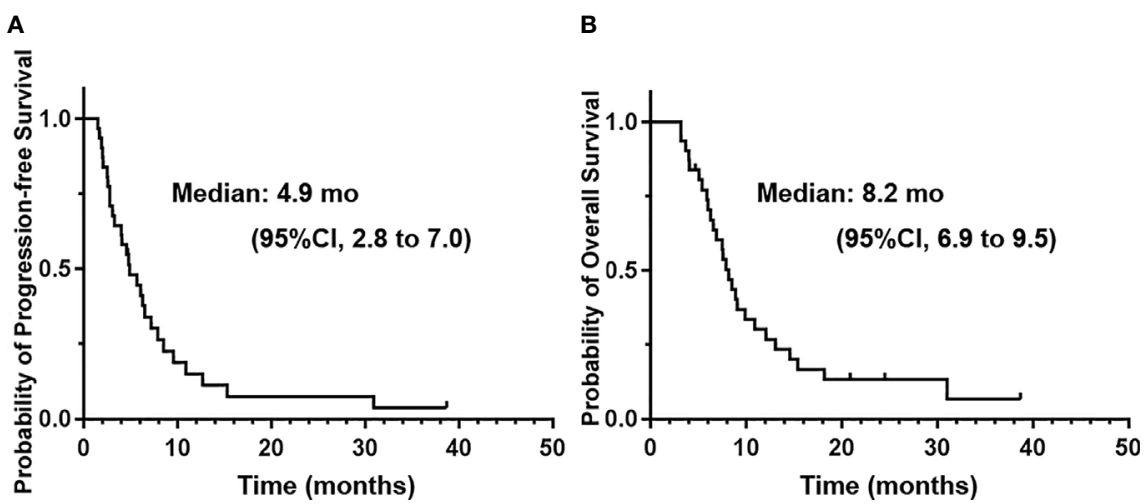

FIGURE 3 | The Kaplan-Meier curves of progression-free survival (A) and overall survival (B) of the whole patients treated with apatinib plus temozolomide.

CI, 6.2 to 10.8 months). The $6 \mathrm{~m}$-PFS and $12 \mathrm{~m}$-OS were 49.4 and $35.1 \%$, respectively.

We also analyzed the relationship between efficacy and MGMT status, number of relapse, tumor dissemination, tumor size, and bevacizumab use after progression (Table 2). The patients without tumor dissemination might have a better prognosis than those with dissemination ( 7.9 versus 4 months for PFS, 12.1 versus 7.5 months for OS). However, there was no statistical difference. After progression, nine out of 28 patients received salvage bevacizumab treatment. The median survival time after progression of the patients with or without bevacizumab was 5.1 (95\%CI, 4.5 to 5.8$)$ versus $1.2(95 \%$ CI, 0 to 2.6) months. The Log-Rank $P=0.174$, while the Breslow $P=0.031$. This indicated that bevacizumab usage after progression may further prolong the survival time. Moreover, the efficacy might be not related to the MGMT status, number of relapse or maximal diameter of tumor, which may be due to the small sample size and need to be confirmed in a largescale study.

\section{Toxicity}

A total of 139 cycles of chemotherapy was available for safety evaluation. Generally, the toxicities of the combined chemotherapy were relatively well tolerated. No one died from drug-related toxicity. Table 3 shows all toxicities representing the sum of the highest grade of toxicities attained from every cycle for all patients.

The most common grade 3 or 4 toxicities were hypertension (5.8\%), decreased appetite (5.8\%), thrombocytopenia (4.3\%), and leukopenia (3.6\%). Most of the grade 3 or 4 toxicities were found in the earlier treatment cycles and could be resolved to grade $\leq 1$ (for non-hematologic toxicities) or grade $\leq 2$ (for hematologic toxicities) after symptomatic treatment, dose interruption or reduction and kept grade $\leq 2$ in the follow-up cycles. Hypothyroidism was found in seven of 12 patients with fatigue. Fatigue was alleviated after treatment with levothyroxine sodium and/or apatinib dose reduction.

Dose reduction occurred in nine patients (eight apatinib, one apatinib and temozolomide) due to fatigue $(6 / 9,66.7 \%)$,

TABLE 2 | Survival time according to potential prognostic factors.

\begin{tabular}{|c|c|c|c|c|c|c|c|}
\hline Prognostic Factor & No. & mPFS (months) ${ }^{a}$ & $6 m-P F S(\%)^{b}$ & $P$-value ${ }^{c}$ & mOS(months) ${ }^{a}$ & $12 \mathrm{~m}-\mathrm{OS}(\%)^{\mathrm{b}}$ & $P$-value ${ }^{c}$ \\
\hline \multicolumn{8}{|l|}{ MGMT status } \\
\hline Unmethylated & 19 & $6.1(4-8.2)$ & $52.6 \pm 11.5$ & 0.791 & $8.5(6.7-10.3)$ & $36.8 \pm 11.1$ & 0.402 \\
\hline Methylated & 5 & $10.9^{d}$ & $60 \pm 21.9$ & & $9.9(5-14.8)$ & $26.7 \pm 22.6$ & \\
\hline \multicolumn{8}{|l|}{ Number of relapse } \\
\hline 1 & 22 & $4.9(3.3-6.5)$ & $44.3 \pm 10.8$ & 0.905 & $8.2(6.7-9.7)$ & $33.6 \pm 10.3$ & 0.997 \\
\hline$>1$ & 9 & $4(2-6)$ & $44.4 \pm 16.6$ & & $7.9(5-10.8)$ & $22.2 \pm 13.9$ & \\
\hline \multicolumn{8}{|l|}{ Tumor dissemination } \\
\hline Yes & 17 & $4(2.7-5.3)$ & $34.3 \pm 11.8$ & 0.1 & $7.5(5.2-9.8)$ & $12.7 \pm 8.4$ & 0.13 \\
\hline No & 9 & $7.9(1.5-14.3)$ & $55.6 \pm 16.6$ & & $12.1(0-25.2)$ & $55.6 \pm 16.6$ & \\
\hline \multicolumn{8}{|l|}{ Maximal diameter } \\
\hline$>30 \mathrm{~mm}$ & 19 & $4.1(1.9-6.3)$ & $35.5 \pm 11.2$ & 0.124 & $7.6(6.2-9)$ & $28.2 \pm 10.6$ & 0.455 \\
\hline$\leq 30 \mathrm{~mm}$ & 12 & $6.1(2.4-9.8)$ & $58.3 \pm 14.2$ & & $8.9(7.9-9.9)$ & $33.3 \pm 13.6$ & \\
\hline \multicolumn{8}{|l|}{ Bev after progression } \\
\hline Yes & 9 & NA & NA & NA & $5.13(4.5-5.8)$ & NA & 0.174 \\
\hline No & 19 & NA & NA & & $1.2(0-2.6)$ & NA & $0.031^{e}$ \\
\hline
\end{tabular}

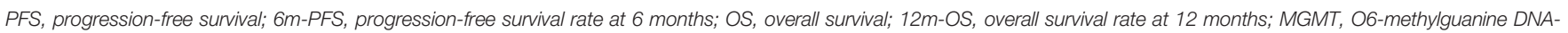
methyltransferase; Bev, bevacizumab; NA, not applicable.

${ }^{a}$ The data were described by median time $(95 \% \mathrm{Cl}) .{ }^{b}$ The data were described by MEAN \pm SEM. ${ }^{c}$ From the log-rank test. ${ }^{d}$ The $95 \%$ CI was not reached. ${ }^{e}$ From the Breslow test. 
TABLE 3 | The toxicities of the combined therapy of apatinib plus temozolomide.

\begin{tabular}{|c|c|c|}
\hline Toxicity $^{a}$ & Any Grade (\%) & Grades 3 and $4(\%)$ \\
\hline \multicolumn{3}{|l|}{ Hematologic } \\
\hline Leukopenia & $58(41.7)$ & $5(3.6)$ \\
\hline Neutropenia & $41(29.5)$ & $4(2.9)$ \\
\hline Lymphocytopenia & $25(18.0)$ & $3(2.2)$ \\
\hline Anemia & $4(2.9)$ & $1(0.7)$ \\
\hline Thrombocytopenia & 36 (25.9) & $6(4.3)$ \\
\hline \multicolumn{3}{|l|}{ Non-hematologic } \\
\hline Hypertension & $60(43.2)$ & $8(5.8)$ \\
\hline Hand-foot syndrome & $19(13.7)$ & $1(0.7)$ \\
\hline Proteinuria & $19(13.7)$ & $2(1.4)$ \\
\hline Fatigue & $28(20.1)$ & $0(0)$ \\
\hline Nausea and vomiting & $4(2.9)$ & $0(0)$ \\
\hline Diarrhea & $11(7.9)$ & $1(0.7)$ \\
\hline Constipation & $4(2.9)$ & $0(0)$ \\
\hline Decreased appetite & $28(20.1)$ & $8(5.8)$ \\
\hline Blood bilirubin increased & $14(10.1)$ & $1(0.7)$ \\
\hline Aminotransferase increased & $24(17.3)$ & $2(1.4)$ \\
\hline LDH increased & $3(2.2)$ & $0(0)$ \\
\hline GGT increased & $6(4.3)$ & $1(0.7)$ \\
\hline Hypokalemia & $5(3.6)$ & $0(0)$ \\
\hline Hyponatremia & $4(2.9)$ & $0(0)$ \\
\hline Hypoalbuminemia & $3(2.2)$ & $0(0)$ \\
\hline ST-T change & $3(2.2)$ & $0(0)$ \\
\hline T wave change & $9(6.5)$ & $0(0)$ \\
\hline Right axis deviation & $2(1.4)$ & $1(0.7)$ \\
\hline Erythra & $4(2.9)$ & $0(0)$ \\
\hline Hoarseness & $5(3.6)$ & $0(0)$ \\
\hline Hypothyroidism & $8(5.8)$ & $0(0)$ \\
\hline Gum bleeding & $1(0.7)$ & $0(0)$ \\
\hline Oral ulcer & $2(1.4)$ & $0(0)$ \\
\hline
\end{tabular}

LDH, lactate dehydrogenase; GGT, gamma-glutamyltransferase.

${ }^{a}$ Ratio of the number of cycles with each toxicity to the total cycles $(n=139)$.

decreased appetite $(3 / 9,33.3 \%)$ and intolerable palpitations (1/ 9, 11.1\%).

\section{DISCUSSION}

We retrospectively analyzed the efficacy of apatinib plus temozolomide in recurrent glioblastoma and observed a high $6 \mathrm{~m}$-PFS rate of $44.6 \%$. The regimen was effective with acceptable toxicities. Moreover, both apatinib and temozolomide are orally administered without the need for hospital admission, meaning that the treatment regimen might have improved adherence and economic effectiveness for patients.

\section{Effect Comparing With Temozolomide Monotherapy}

In this study, the combined regimen could have a superior efficacy to temozolomide monotherapy. Clinical studies demonstrated that the $6 \mathrm{~m}$-PFS of the patients with recurrent glioblastoma who received temozolomide on standard 5 days, 7 days on/7 days off or continuous dose-intense schedules was 21, 10 and 23.9\%, respectively (17-19). In the DERECTOR trial, 105 patients with glioblastoma at first progression was enrolled and received dose-intensified temozolomide treatment (20). The median PFS and $6 \mathrm{~m}$-PFS for 7 days on/7days off regimen were 1.8 months and $17.1 \%$, while that for 3 weeks on/one week off regimen were 2.0 months and $25 \%$.

However, in our study, the median PFS of the whole patients was 4.9 months and the $6 \mathrm{~m}$-PFS was $44.6 \%$, which were better that that of temozolomide. Moreover, two patients had long PFS of 30.9 and $38.7+$ months, surpassing their prior recurrence intervals of 12 and 14 months, respectively. These suggested that apatinib was likely to enhance the effect of temozolomide, which is consistent with the results of several preclinical studies, which have demonstrated that inhibiting VEGFR-2 could enhance the efficacy of temozolomide. In vitro, down-regulated VEGFR-2 results in decreased cell proliferation and higher sensitivity of glioma cells to temozolomide-induced G2 cell cycle arrest (21). In an intracranial murine xenograft model, inhibiting VEGFR-2 suppressed glioblastoma growth and prolonged mouse survival time, both of which were augmented by the incorporation of temozolomide (22).

\section{Effect Comparing With Bevacizumab- Based Therapy}

Table 4 lists the main study of bevacizumab in recurrent glioblastoma in recent years $(4-6,23,24)$. A phase II randomized non-comparative trial of bevacizumab with or without irinotecan in recurrent glioblastoma participants in first or second relapse revealed that the ORR was $28-38 \%$ and $6 \mathrm{~m}$-PFS was $43-50 \%$. Median OS was 9.2 months in the bevacizumab monotherapy arm and 8.7 months in the combination arm (4). The FDA reviewed and determined that the ORR of bevacizumab was $26 \%$ and the $6 \mathrm{~m}$-PFS was $36 \%$. In the EORTC 26101 phase 3 trial in patients with first relapsed glioblastoma, the $6 \mathrm{~m}$-PFS of the bevacizumab plus lomustine group was $30.2 \%$, the median PFS was 4.2 months and median OS was 9.1 months (6). In our study, the ORR was $26.3 \%$, the median PFS was 4.9 months and $6 \mathrm{~m}$-PFS was $44.6 \%$. This indicated that the effect of apatinib plus temozolomide was comparable to that of bevacizumab.

TABLE 4 | Overview of bevacizumab study in recurrent glioblastoma in recent years.

\begin{tabular}{|c|c|c|c|c|c|c|}
\hline Year & Author & Agents & Patients No. & Relapse No. & $6 m-P F S$ & OS (months) \\
\hline \multirow[t]{2}{*}{2009 (4) } & Friedman HS & Bev; & 85 & First or second & $42.6 \%$ & 9.2 \\
\hline & & $\mathrm{Bev}+|\mathrm{R}|$ & 82 & & $50.3 \%$ & 8.7 \\
\hline 2009 (5) & Kreisl TN & Bev & 48 & First & $29 \%$ & 7.1 \\
\hline 2012 (23) & Desjardins A & $\mathrm{Bev}+\mathrm{TMZ}$ & 32 & First or second or third & $18.8 \%$ & 8.5 \\
\hline \multirow[t]{2}{*}{$2014(24)$} & Taal W & Bev; & 50 & first & $16 \%$ & 8 \\
\hline & & $\mathrm{Bev}+\mathrm{CCNU}$ & 52 & & $42 \%$ & 12 \\
\hline 2017 (6) & Wick W & $\mathrm{Bev}+\mathrm{CCNU}$ & 288 & First & $30.2 \%$ & 9.1 \\
\hline Current study & Ge JJ & Apatinib + TMZ & 31 & First or second or third & $44.6 \%$ & 8.2 \\
\hline
\end{tabular}

Bev, bevacizumab; IRI, irinotecan; CCNU, lomustine; TMZ, temozolomide; $6 m-P F S$, progression-free survival rate at 6 months; OS, overall survival. 
Moreover, the small molecule apatinib has some characteristics that differ from bevacizumab. Firstly, apatinib is orally administered and convenient to take while bevacizumab is administered intravenously in a clinic or hospital. Secondly, the short half-life of apatinib ( $9 \mathrm{~h}$ for apatinib versus 3 weeks for bevacizumab) allows for quick normalization of wound healing in case of urgent surgical intervention (25). In bevacizumab, however, surgery should not be performed for at least 28 days after the last bevacizumab administration.

\section{Salvage Treatment After Tumor Progression}

Our study observed that, after tumor progression from the combined chemotherapy, bevacizumab usage could help to prolong the survival time (5.1 versus 1.2 months). This suggested that the current combined regimen did not reduce the sensitivity of tumor to bevacizumab.

Furthermore, in our study, the median time interval between disease progressions to death was 3.2 months, which is shorter than other reports. A possible explanation for this is that only 15 of 29 patients received anti-tumor therapy after tumor progression, including resection, bevacizumab or other antiangiogenic agents.

\section{Efficacy Based on Genetic Characterization}

MGMT promoter methylation is a prognostic factor. Recurrent glioblastoma with unmethylated MGMT has a poor prognosis (20). After treatment with dose-intensified temozolomide, the PFS was only 1.8 months and the 6m-PFS was $6.9 \%$ (20). Even treating with bevacizumab and lomustine, the PFS was 3.02 months and the $6 \mathrm{~m}$-PFS was $12.7 \%$ (6). In contrast with these reports, we observed a superior PFS of 6.1 months and 6m-PFS of 52.6\% for the 19 recurrent glioblastoma with unmethylated MGMT.

IDH mutation status is also associated with prognosis. In our study, 20 patients harbored wild-type IDH, 15 of which were also MGMT unmethylated. For all the 20 patients with wild-type IDH, the median PFS and 6m-PFS were 5.7 months and $48.7 \%$. Wang Y et al. reported that, after treatment of apatinib plus temozolomide, 19 recurrent glioblastoma with wild-type IDH got a PFS of 5.9 months and $6 \mathrm{~m}$-PFS of $47.4 \%$, which was similar to our study (26). However, in that study, there was no information about the MGMT methylation status, number of relapse, or tumor dissemination.

\section{CONCLUSION}

The current study preliminarily shows that the combined therapy of apatinib and temozolomide (1) has a promising efficacy, which could be better than that of temozolomide alone, especially for the patients with unmethylated MGMT

\section{REFERENCES}

1. Zuniga RM, Torcuator R, Jain R, Anderson J, Doyle T, Ellika S, et al. Efficacy, safety and patterns of response and recurrence in patients with recurrent high-grade gliomas treated with bevacizumab plus irinotecan. J Neurooncol (2009) 91:329-36. doi: 10.1007/s11060-008-9718-y and wild-type IDH; (2) does not reduce the sensitivity of tumor to bevacizumab; (3) has manageable toxicities; (4) is orally administered without the need for hospital admission. The major limitations of this study are retrospective design and the relatively small sample size. A randomized controlled trial should be conducted to provide more definitive evidence.

\section{DATA AVAILABILITY STATEMENT}

The raw data supporting the conclusions of this article will be made available by the authors, without undue reservation.

\section{ETHICS STATEMENT}

The studies involving human participants were reviewed and approved by the Ethics Committee of Sanbo Brain Hospital Capital Medical University. The patients/participants provided their written informed consent to participate in this study. Written informed consent was obtained from the individual(s) for the publication of any potentially identifiable images or data included in this article.

\section{AUTHOR CONTRIBUTIONS}

JG: Acquisition of data, analysis and drafting, and revising the article. CL, FX, SQ: Acquisition of data, analysis and interpretation of data. ZG: Acquisition of data. CY: Conception and design. JZ: Conception and design of the work and revision of the article. All authors contributed to the article and approved the submitted version.

\section{FUNDING}

This work was supported by Scientific Research Common Program of Beijing Municipal Commission of Education (Grant number: KM201710025027) and Neuro-oncology Research Program of Chinese Society of Neuro-oncology (Grant number: CSNO-2014-MSD02).

\section{ACKNOWLEDGMENTS}

The authors wish to thank patients and their caregivers for participating in this study.

2. Stupp R, Mason WP, van den Bent MJ, Weller M, Fisher B, Taphoorn MJ, et al. Radiotherapy plus concomitant and adjuvant temozolomide for glioblastoma. N Engl J Med (2005) 352:987-96. doi: 10.1056/ NEJMoa043330

3. Louis DN, Perry A, Reifenberger G, von Deimling A, Figarella-Branger D, Cavenee WK, et al. The 2016 World Health Organization Classification of 
Tumors of the Central Nervous System: a summary. Acta Neuropathol (2016) 131:803-20. doi: 10.1007/s00401-016-1545-1

4. Friedman HS, Prados MD, Wen PY, Mikkelsen T, Schiff D, Abrey LE, et al. Bevacizumab alone and in combination with irinotecan in recurrent glioblastoma. J Clin Oncol (2009) 27:4733-40. doi: 10.1200/JCO.2008.19.8721

5. Kreisl TN, Kim L, Moore K, Duic P, Royce C, Stroud I, et al. Phase II trial of single-agent bevacizumab followed by bevacizumab plus irinotecan at tumor progression in recurrent glioblastoma. J Clin Oncol (2009) 27:740-5. doi: 10.1200/JCO.2008.16.3055

6. Wick W, Gorlia T, Bendszus M, Taphoorn M, Sahm F, Harting I, et al. Lomustine and Bevacizumab in Progressive Glioblastoma. N Engl J Med (2017) 377:1954-63. doi: 10.1056/NEJMoa1707358

7. Tipping M, Eickhoff J, Ian Robins H. Clinical outcomes in recurrent glioblastoma with bevacizumab therapy: An analysis of the literature. J Clin Neurosci (2017) 44:101-6. doi: 10.1016/j.jocn.2017.06.070

8. Ferrara N. The role of VEGF in the regulation of physiological and pathological angiogenesis. EXS (2005) 94):209-31. doi: 10.1007/3-7643-7311-3_15

9. Li J, Qin S, Xu J, Xiong J, Wu C, Bai Y, et al. Randomized, Double-Blind, PlaceboControlled Phase III Trial of Apatinib in Patients With Chemotherapy-Refractory Advanced or Metastatic Adenocarcinoma of the Stomach or Gastroesophageal Junction. J Clin Oncol (2016) 34:1448-54. doi: 10.1200/JCO.2015.63.5995

10. Lu W, Jin XL, Yang C, Du P, Jiang FQ, Ma JP, et al. Comparison of efficacy between TACE combined with apatinib and TACE alone in the treatment of intermediate and advanced hepatocellular carcinoma: A single-center randomized controlled trial. Cancer Biol Ther (2017) 18(6):433-8. doi: 10.1080/15384047.2017.1323589

11. Lan CY, Wang Y, Xiong Y, Li JD, Shen JX, Li YF, et al. Apatinib combined with oral etoposide in patients with platinum-resistant or platinum-refractory ovarian cancer (AEROC): a phase 2, single-arm, prospective study. Lancet Oncol (2018) 19(9):1239-46. doi: 10.1016/S1470-2045(18)30349-8

12. Wu D, Liang L, Nie L, Nie J, Dai L, Hu W, et al. Efficacy, safety and predictive indicators of apatinib after multilines treatment in advanced nonsquamous nonsmall cell lung cancer: Apatinib treatment in nonsquamous NSCLC. Asia Pac J Clin Oncol (2018) 14:446-52. doi: 10.1111/ajco.12870

13. Tong XZ, Wang F, Liang S, Zhang X, He JH, Chen XG, et al. Apatinib (YN968D1) enhances the efficacy of conventional chemotherapeutical drugs in side population cells and ABCB1-overexpressing leukemia cells. Biochem Pharmacol (2012) 83:586-97. doi: 10.1016/j.bcp.2011.12.007

14. Mi YJ, Liang YJ, Huang HB, Zhao HY, Wu CP, Wang F, et al. Apatinib (YN968D1) reverses multidrug resistance by inhibiting the efflux function of multiple ATP-binding cassette transporters. Cancer Res (2010) 70:7981-91. doi: 10.1158/0008-5472.CAN-10-0111

15. Wang C, Jiang M, Hou H, Lin Q, Yan Z, Zhang X. Apatinib suppresses cell growth and metastasis and promotes antitumor activity of temozolomide in glioma. Oncol Lett (2018) 16:5607-14. doi: 10.3892/ol.2018.9355

16. Wen PY, Macdonald DR, Reardon DA, Cloughesy TF, Sorensen AG, Galanis E, et al. Updated response assessment criteria for high-grade gliomas: response assessment in neuro-oncology working group. J Clin Oncol (2010) 28:1963-72. doi: 10.1200/JCO.2009.26.3541

17. Perry JR, Bélanger K, Mason WP, Fulton D, Kavan P, Easaw J, et al. Phase II trial of continuous dose-intense temozolomide in recurrent malignant glioma: RESCUE study [published correction appears in J Clin Oncol. 2010 Jul 20;28(21):3543]. J Clin Oncol (2010) 28:2051-7. doi: 10.1200/ JCO.2009.26.5520

18. Yung WK, Albright RE, Olson J, Fredericks R, Fink K, Prados MD, et al. A phase II study of temozolomide vs. procarbazine in patients with glioblastoma multiforme at first relapse. Br J Cancer (2000) 83:588-93. doi: 10.1054/ bjoc. 2000.1316

19. Han SJ, Rolston JD, Molinaro AM, Clarke JL, Prados MD, Chang SM, et al. Phase II trial of 7 days on/7 days off temozolmide for recurrent high-grade glioma. Neuro Oncol (2014) 16:1255-62. doi: 10.1093/neuonc/nou044

20. Weller M, Tabatabai G, Kästner B, Felsberg J, Steinbach JP, Wick A, et al. MGMT Promoter Methylation Is a Strong Prognostic Biomarker for Benefit from Dose-Intensified Temozolomide Rechallenge in Progressive Glioblastoma: The DIRECTOR Trial. Clin Cancer Res (2015) 21:2057-64. doi: 10.1158/1078-0432.CCR-14-2737

21. Kessler T, Sahm F, Blaes J, Osswald M, Rübmann P, Milford D, et al. Glioma cell VEGFR-2 confers resistance to chemotherapeutic and antiangiogenic treatments in PTEN-deficient glioblastoma. Oncotarget (2015) 6:31050-68. doi: 10.18632 /oncotarget.2910

22. Waters JD, Sanchez C, Sahin A, Futalan D, Gonda DD, Scheer JK, et al. CT322, a VEGFR-2 antagonist, demonstrates anti-glioma efficacy in orthotopic brain tumor model as a single agent or in combination with temozolomide and radiation therapy. J Neurooncol (2012) 110:37-48. doi: 10.1007/s11060-012-0948-7

23. Desjardins A, Reardon DA, Coan A, Marcello J, Herndon JE2, Bailey L, et al. Bevacizumab and daily temozolomide for recurrent glioblastoma. Cancer (2012) 118(5):1302-12. doi: 10.1002/cncr.26381

24. Taal W, Oosterkamp HM, Walenkamp AM, Dubbink HJ, Beerepoot LV, Hanse MC, et al. Single-agent bevacizumab or lomustine versus a combination of bevacizumab plus lomustine in patients with recurrent glioblastoma (BELOB trial): a randomised controlled phase 2 trial. Lancet Onco (2014) 15(9):943-53. doi: 10.1016/S1470-2045(14)70314-6

25. Li J, Zhao X, Chen L, Guo H, Lv F, Jia K, et al. Safety and pharmacokinetics of novel selective vascular endothelial growth factor receptor-2 inhibitor YN968D1 in patients with advanced malignancies. BMC Cancer (2010) 10:529. doi: 10.1186/1471-2407-10-529

26. Wang Y, Meng X, Zhou S, Zhu Y, Xu J, Tao R. Apatinib Plus Temozolomide for Recurrent Glioblastoma: An Uncontrolled, Open-Label Study. Onco Targets Ther (2019) 12:10579-85. doi: 10.2147/OTT.S226804

Conflict of Interest: The authors declare that the research was conducted in the absence of any commercial or financial relationships that could be construed as a potential conflict of interest.

Copyright $\odot 2021 \mathrm{Ge}, \mathrm{Li}, \mathrm{Xue}, \mathrm{Qi}, \mathrm{Gao}, \mathrm{Yu}$ and Zhang. This is an open-access article distributed under the terms of the Creative Commons Attribution License (CC BY). The use, distribution or reproduction in other forums is permitted, provided the original author(s) and the copyright owner(s) are credited and that the original publication in this journal is cited, in accordance with accepted academic practice. No use, distribution or reproduction is permitted which does not comply with these terms. 